\title{
Modeling the spectrum of TT Hya: an Algol binary with a disc
}

\author{
J. Budaj ${ }^{1,2}$ and M.T. Richards ${ }^{1}$ \\ ${ }^{1}$ Dept. of Astronomy and Astrophysics, Penn State University, Davey Lab. 525, University \\ Park, PA 16802, USA, email: budaj@astro.psu.edu \\ ${ }^{2}$ Astronomical Institute, Tatranská Lomnica, 05960, Slovakia
}

\begin{abstract}
We calculate the synthetic spectra of TT Hya, an Algol-type eclipsing binary system which contains an A-type primary surrounded by a disc, and an evolved secondary which fills its Roche lobe. A new code SHELLSPEC was developed to solve the simple radiative transfer along the line of sight propagating through the 3D moving medium in LTE. The synthetic spectra are then compared with the observed ones which enable us to derive independent constraints on the behavior of the state quantities, the velocity field and the geometry of the disc.
\end{abstract}

Keywords. Radiative transfer, accretion, accretion discs, stars: binaries: eclipsing, stars: binaries: close, stars: novae, cataclysmic variables, stars: individual: (TT Hya)

\section{Introduction}

There are sophisticated computer codes for calculating and inverting light curves of spectra of binary stars with various shapes or geometry including the Roche model (Lucy 1968, Wilson \& Devinney 1971, Mochnacki \& Doughty 1972, Rucinski 1973, Hill 1979, Zhang et al. 1986, Drechsel et al. 1994, Vinkó et al. 1996, Djurasevic 1992, Hadrava 1997, Bradstreet \& Steelman 2002, Pribulla 2004). In these codes, the stars are assumed to be nontransparent, stripped of any circumstellar matter and their main purpose is to deal with the complicated geometry. However, it is often the case that such nontransparent objects are embedded in some moving optically thin environment (Cherepashchuk et al. 1984) and/or are accompanied by shells, discs, jets or streams and one needs to know at least an approximate spectrum, light curve and trailed spectrogram. Often the 3D model (behavior of the state quantities and the velocity field) is known or expected as a result of hydrodynamic simulations or observational constraints (see, e.g., Richards \& Ratliff 1998). Unfortunately, 3D NLTE calculations including complex hydrodynamics are difficult to carry out so one alternative has been to perform a simple volume integration of emissivity, which is often too oversimplified for the particular problem.

On the other hand, highly sophisticated model atmospheres and spectrum synthesis codes were developed assuming NLTE and plane-parallel atmospheres of hot stars (Hubeny 1988, Hubeny \& Lanz 1992, 1995, Hubeny et al. 1994), spherically symmetric atmospheres (Kubát 2001), or stellar winds (Krtička \& Kubát 2002). There are also sophisticated stationary plane-parallel line-blanketed model atmospheres and spectrum synthesis codes for a large variety of stars assuming LTE (Kurucz 1990, 1993, Smith \& Dworetsky 1988, Piskunov 1992, and many others). However, these are very specialized codes and their main purpose is to calculate the spectrum emerging from a stellar atmosphere and it is difficult to apply them to the various cases outlined above. An exception is the special case of circumstellar matter in the form of accretion discs in CV's. In this case, the disc can be approximated by a set of geometrically thin, but optically thick, static local atmospheres and the output radiation is a sum of properly Doppler shifted 
local emerging intensities (Orosz \& Wade 2003, Wade \& Hubeny 1998, la Dous 1989). For optically thin discs or accretion disc winds, the Sobolev approximation is used (Proga et al. 2002, Long \& Knigge 2002, Rybicki \& Hummer 1983). Horne \& Marsh (1986) solved the radiative transfer along the line of sight in a moving disc and showed that it might be important.

The goal of this paper is not to compete with the codes and models mentioned above but rather to bridge the gap in these present approaches and provide a tool which would solve in LTE the simple radiative transfer along the line of sight in an optional optically thin 3D moving medium with possible nontransparent objects embedded in. We present the new code to synthesize the composite spectrum of accretion structures in close binaries which undergo direct impact accretion, as in the close Algols. However, the code is a multi-purpose, independent, and flexible tool which can also calculate a light curve or a trailed spectrogram where necessary, or can be used to study various objects or effects.

\section{Basic astrophysics}

\subsection{Radiative transfer}

The calculations are carried out in the observer's Cartesian frame. The radiative transfer equation along the line of sight is solved assuming the following opacities and emissivities:

-the line opacity via a shifted Voigt profile which includes the contributions from the Natural, Stark, Van der Waals and thermal broadening and microturbulence

-the continuum opacity sources: H I bound-free opacity, H I free-free opacity, Thomson scattering, Rayleigh scattering on neutral hydrogen

- the thermal emissivity associated with the true absorption

-the scattering emissivity from the central object.

The equation of the radiative transfer along the line of sight at the frequency $\nu$ is written in the discretised form, and integrated via the simple recurrent formula.

If the line of sight happens to hit a nontransparent object, the object is skipped and the integration starts on the other side of the object with the appropriate boundary condition. If the line of sight happens to pass through an empty space this region is also skipped and the integration continues with the unchanged intensity. The flux from the object at the Earth is then obtained by the integration of the output intensities through the $2 \mathrm{D}$ projection surface of the $3 \mathrm{D}$ object.

\subsection{Roche geometry}

Both objects, STAR and COMPANION may have the shape according to the Roche model for detached or contact systems. Gravity darkening is taken into account by varying the surface temperature according to the gravity darkening law. Limb darkening and the reflection effect of objects subject to Roche geometry are not included in the present version. However, limb darkening is included for spherical objects.

\subsection{Rotation of the observer's frame}

The SHELLSPEC code enables the user to look at the object from different points of view and to calculate the corresponding spectra. The input model is defined in its body frozen Cartesian coordinates $\left(x^{\prime \prime}, y^{\prime \prime}, z^{\prime \prime}\right)$ with the $z^{\prime \prime}$ axis corresponding to the intrinsic rotation axis of the model. The spectrum is always calculated in the observer's line of 
sight Cartesian frame $(x, y, z)$ with z pointing to the observer and which has the same center of coordinates.

\subsection{Level populations and miscellaneous notes}

The code assumes the known behavior of the state quantities: temperature, density, and electron number density. The atomic number density of all atoms is calculated from the density, element abundances and atomic masses. The hydrogen number density is calculated from the atomic number density. Element number densities are calculated from the abundances and the hydrogen number density. Solar abundances are assumed but the user can change the elemental abundances. The level populations are obtained from the Boltzmann and Saha equations. Within the code, cgs units are used, if not specified otherwise.

\subsection{Adopted routines}

Several routines used in this code were adopted from other sources. These are: pfdwor (from UCLSYN, Smith \& Dworetsky 1988); voigt0, state0, gaunt, gfree (from SYNSPEC, Hubeny et al. 1994); and locate, hunt (from Numerical Recipes, Press et al. 1986). We also used a few sections from our earlier original codes for calculations of radiative accelerations in stellar atmospheres of hot stars (Budaj \& Dworetsky 2002). Apart from the routines mentioned above, the code was written from scratch and provides an independent tool to study a large variety of objects and effects.

\section{Input models}

In the main input file you describe the geometry, dynamics and optical properties of the moving medium. However, it can consist of many objects of various types. You can also load a precalculated model from an extra file. Various transparent and nontransparent objects can be defined here:

STAR: a central nontransparent object which can rotate as a solid body with an optional inclination of the rotational axis and have a net space velocity. Can be treated as a blackbody or have its own spectrum. Limb darkening can be applied to it. Also, the scattered light from this object can be taken into account (without rotation and limb darkening). Object can be surrounded by a Keplerian disc which is specified separately. Designed to model mainly hotter or more luminous stellar components.

COMPANION: a nontransparent object which can rotate as a solid body with an optional inclination of the rotational axis and have a net space velocity. Limb darkening can be applied to it. Can be treated as a blackbody or have its own spectrum. Designed to model mainly a secondary (cooler or fainter) component of a binary system.

SPOT: a spherical object which can rotate with an optional inclination of the rotational axis and have a net space velocity. Designed to model mainly spots on accretion discs, direct impact regions or rotating circumstellar (or circumbinary) shells or third bodies. DISC: has either the shape of a space complement to two opposite cones or of a cylinder surrounding the central object. It is constrained by its inner and outer radius. This structure can be inclined if necessary. The velocity of the disc is Keplerian and depends on the mass of the central object. It is possible to vary the densities as a power law or adopt a temperature stratification if necessary. Designed to model mainly accretion discs. STREAM: has the shape of a cylinder with uniform velocity. Designed to model a mass transfer stream.

JET: has the shape of one or two opposite cones emerging from the center. It can have an optional inclination and is farther limited by its inner and outer radii. Designed to model 


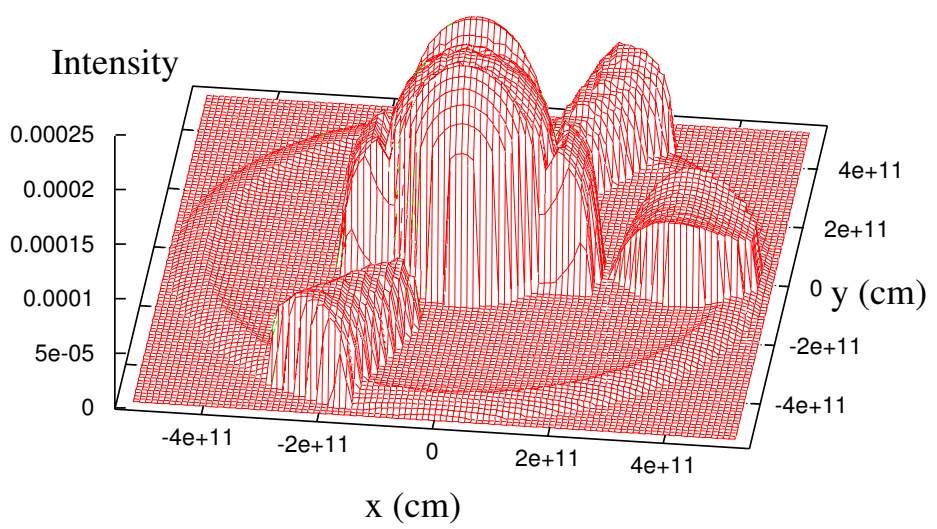

Figure 1. 2D projection image, $I_{\nu}^{\text {out }}(x, y)$, of a test model at one particular phase.

mainly jets or, e.g., shadows cast by a cool, extended secondary from a more compact hot primary.

SHELL: has the shape of a shell surrounding the central object. Various velocity fields are built in.

BACKGROUND: is designed to add more flexibility to the code and to fill the region not occupied by any of the previous objects at least with a uniformly radially expanding medium when necessary.

\section{Demonstration of an artificial model}

As an illustration, we include a few pictures calculated for an artificial spectral line and an artificial test model. It includes two stars, a Keplerian equatorial disc around a larger primary, a slightly inclined jet and a slowly expanding shell surrounding the system. Stars are treated as blackbodies, the primary is a sphere with limb darkening while the secondary fills its Roche lobe and is subject to gravity darkening only. The centers of the jets and shell have no net space velocity while the net velocity of the center of the disc corresponds to that of the primary. Calculations were performed for about 50 phases as seen from the orbital plane. The model was defined in a cube with 101x101x101 points and spectrum was calculated at 241 frequency points. For the purpose of this illustration, the input values were manipulated so that a contribution from each object could be seen. Figure 1 shows a 2D projection image of a test model in the continuum (at a frequency in the far wing of the spectral line) taken roughly at the quadrature. Different shapes of the primary and secondary illustrate the effects of limb and gravity darkening. Figure 2 shows the overall light curve of the model as it revolves. The primary and secondary minima can be easily identified there. Figure 3 displays the trailed spectrogram. The double wave is caused by the two jet cones while the single wave is caused by the orbital motion of the disc tracing that of the primary. Observe two depressions in S-waves adjacent to the 


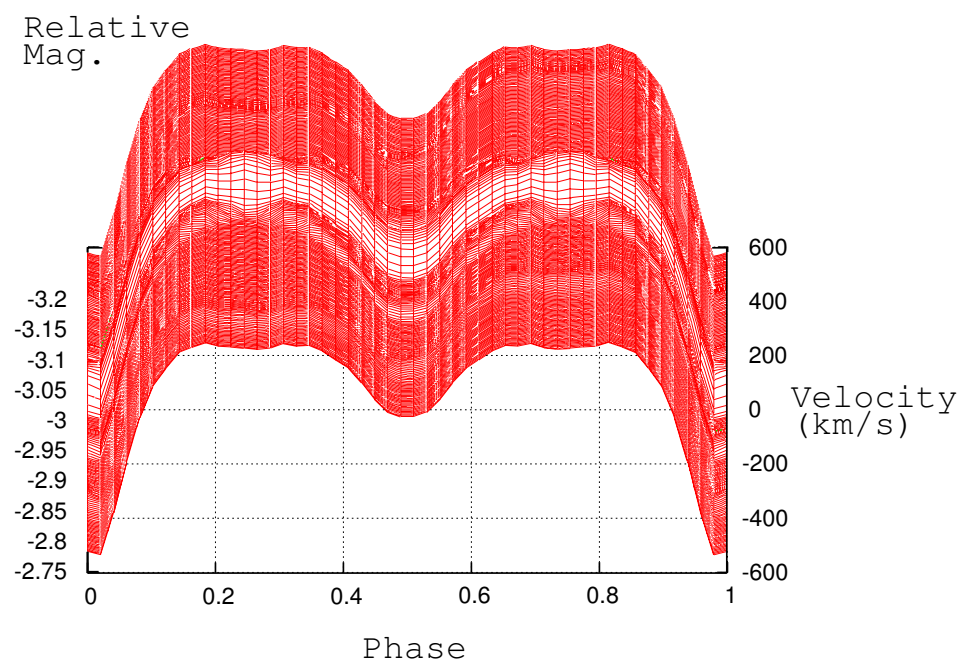

Figure 2. Overall light curve of the test model.

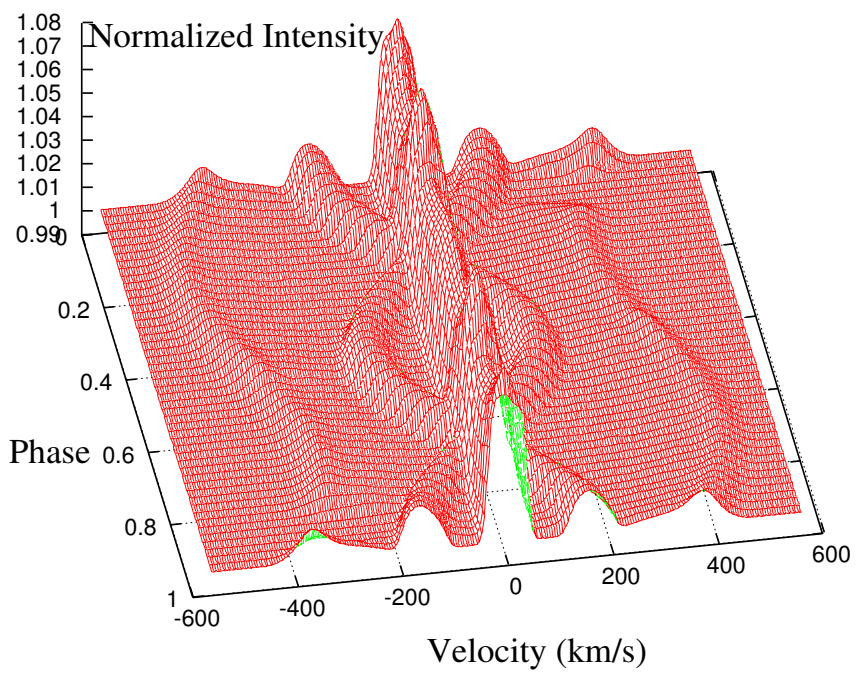

Figure 3. Trailed spectrogram of the test model.

primary eclipse caused by the eclipse of the receding and approaching part of the disc, respectively. The central emission comes from the shell. 


\section{Application to accretion disc systems like TT Hya}

Another and more realistic test of the code was to calculate synthetic spectra of $H \alpha$ with a geometry similar to the well-known Algol-type binary TT Hya. The system exhibits the emission features which were ascribed to the disc (Plavec \& Polidan 1976) surrounding the primary. It has been studied by Kulkarni \& Abhyankar (1980), Plavec (1988), Peters (1989), Van Hamme \& Wilson (1993), Albright \& Richards (1996), Peters \& Polidan (1998), and Richards \& Albright (1999). The secondary contributes little to the spectrum. We first investigate how the emerging spectrum responds to the variation of the different input parameters. The calculations were performed with the relatively low xyz resolution of $76 \times 76 \times 76$ points for the region containing the primary and the disc. The primary was treated as a blackbody with the following parameters: effective temperature $T_{\text {eff }}=$ $9800 \mathrm{~K}$, mass $M=2.25 M_{\odot}$, radius $R=1.9 R_{\odot}$ (Etzel 1988). The disc was characterized by inner radius $R_{\text {in }}=2.5 R_{\odot}$, outer radius $R_{\text {out }}=10 R_{\odot}$, angular half-width $\alpha=15^{\circ}$, electron number density $n_{e}=3 \times 10^{9} \mathrm{~cm}^{-3}$, density $\rho=5 \times 10^{-15} \mathrm{~g} \mathrm{~cm}^{-3}$, exponent of the density power law behaviour edendc $=0$., temperature $T=7000 \mathrm{~K}$, microturbulence $v_{\text {turb }}=0 \mathrm{~km} \mathrm{~s}^{-1}$ and inclination $i=84.4^{\circ}$. Figures $4,5,6,7,8,9,10,11,12$, and 13 illustrate the effects of varying one of the free parameters of the system at a time. In reality, the primary star (as well as the secondary) is not a blackbody but has its own spectrum where the $H \alpha$ line is not negligible and in fact dominates the optical spectrum of Algol-type binaries. So, we calculated the synthetic spectrum of the primary with the code SYNSPEC (Hubeny et al. 1994, Krtička 1998). This spectrum was then assigned to the primary and a more complex spectrum of the disc and primary was calculated using SHELLSPEC and depicted in Figure 14. We assumed solar abundances and the most extreme case of zero rotational velocity of the primary. Figure 15 displays the synthetic difference spectra calculated under the assumption that the primary is either a blackbody or has its own synthetic spectrum (precalculated with SYNSPEC). There is no significant difference in the difference profiles except in the central depression. Finally, we tried to fit the observed difference profiles of the system obtained by Richards \& Albright (1999) at the quadrature. A good fit of the observations was achieved with the reasonable parameters of the disc and primary which are summarized in the Table 1. The primary was treated as a blackbody. Figure 16 shows both the observed difference profile and our synthetic difference profile.

Table 1. Properties of TT Hya.

\begin{tabular}{ll}
\hline Physical properties of the primary: & \\
$M^{\star}$ & $2.25 M_{\odot}$ \\
$T^{\star}$ & $9800 \mathrm{~K}$ \\
$R^{\star}$ & $1.9 R_{\odot}$ \\
limb darkening coeff. & 0.5 \\
& \\
Assumed properties of the disc: & \\
$i$ & $84.4^{\circ}$ \\
$\alpha$ & $15^{\circ}$ \\
$R_{\text {in }}$ & $1.9 R_{\odot}$ \\
$R_{\text {out }}$ & $10 R_{\odot}$ \\
$\rho\left(R_{\text {in }}\right)$ & $78 \times 10^{-15} \mathrm{~g} \mathrm{~cm}^{-3}$ \\
$n e\left(R_{\text {in }}\right)$ & $46 \times 10^{9} \mathrm{~cm}^{-3}$ \\
edendc & -2 \\
$v_{\text {turb }}$ & $40 \mathrm{~km} \mathrm{~s}^{-1}$ \\
\hline
\end{tabular}




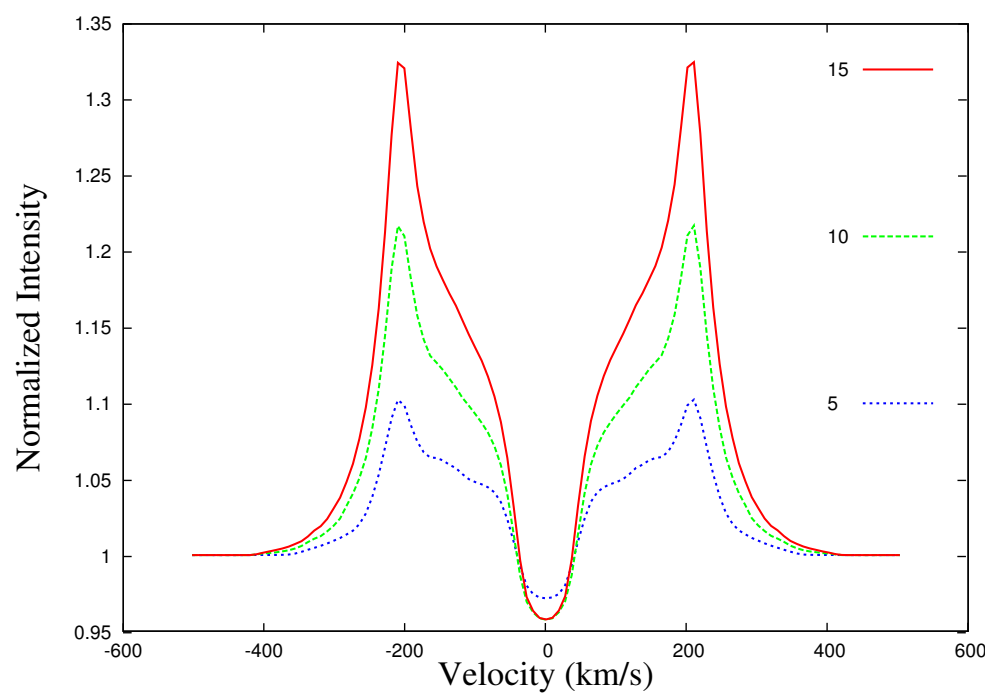

Figure 4. Effect of varying alpha (angular half-width of the disc) from 5 to 15 degrees.

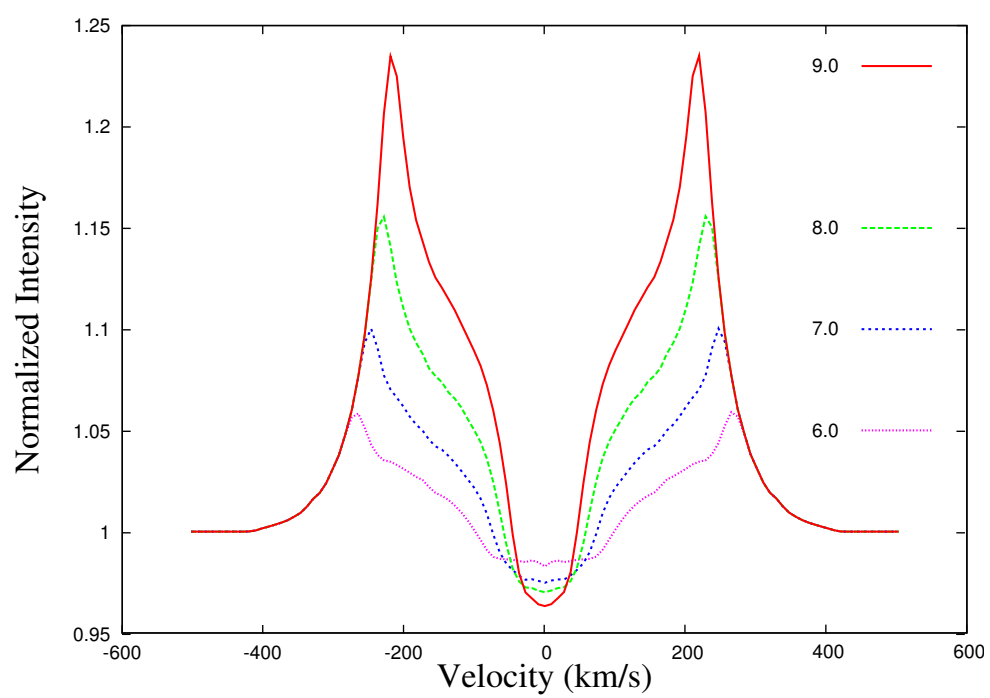

Figure 5. Effect of varying the outer radius of the disc from 6 to $9 R_{\odot}$. 


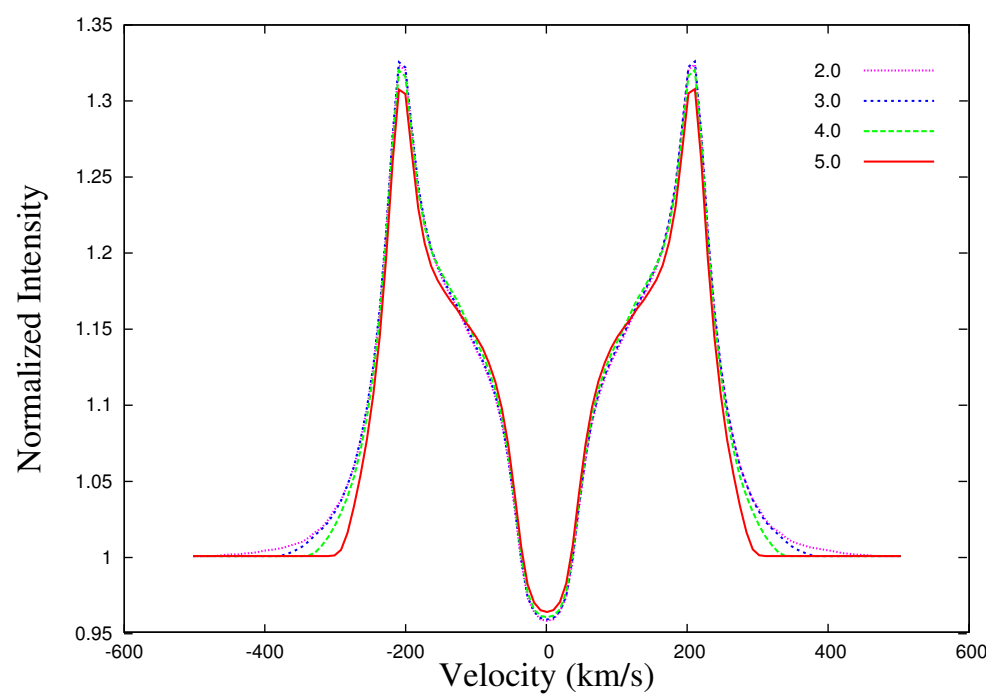

Figure 6. Effect of varying the inner radius of the disc from 2 to $5 R_{\odot}$.

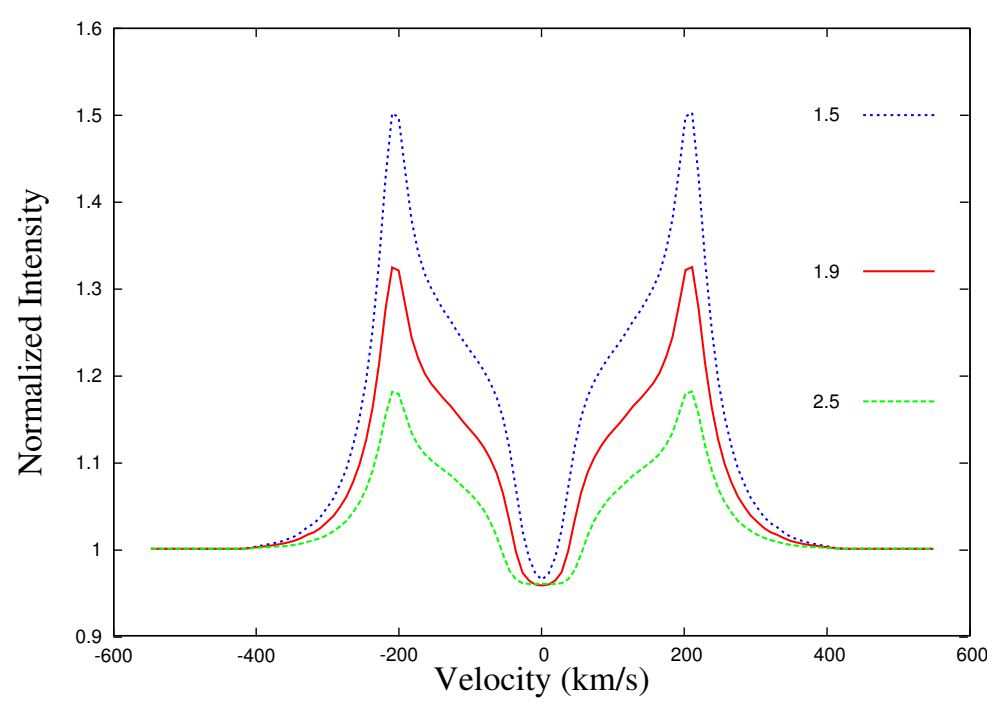

Figure 7. Effect of varying the radius of the star from 1.5 to $2.5 R_{\odot}$. 


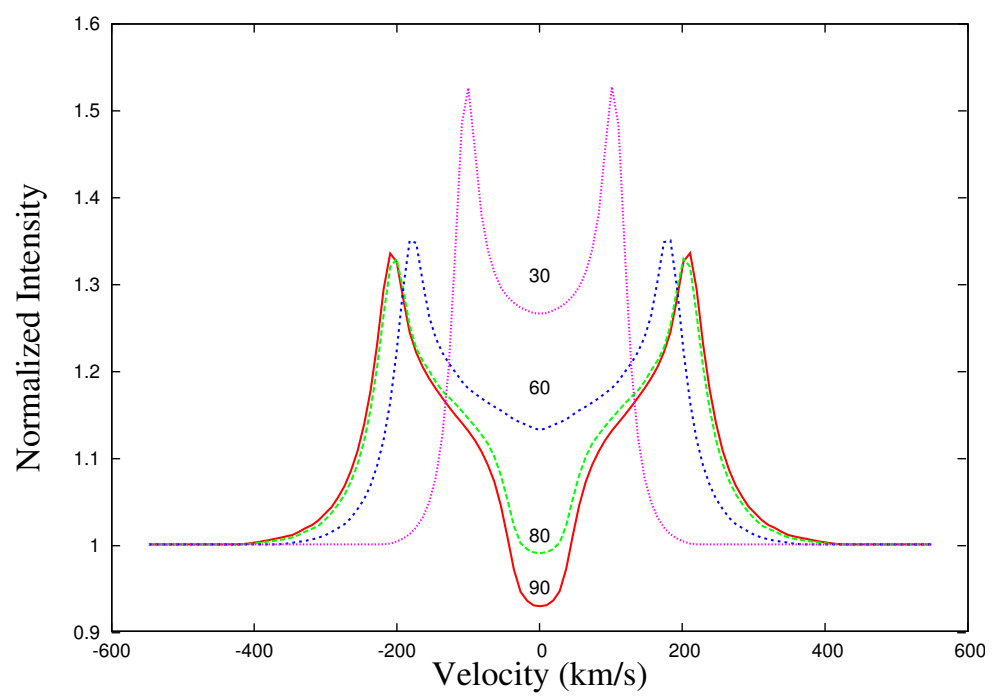

Figure 8. Effect of changing the inclination of the disc from $90^{\circ}$ to $30^{\circ}$.

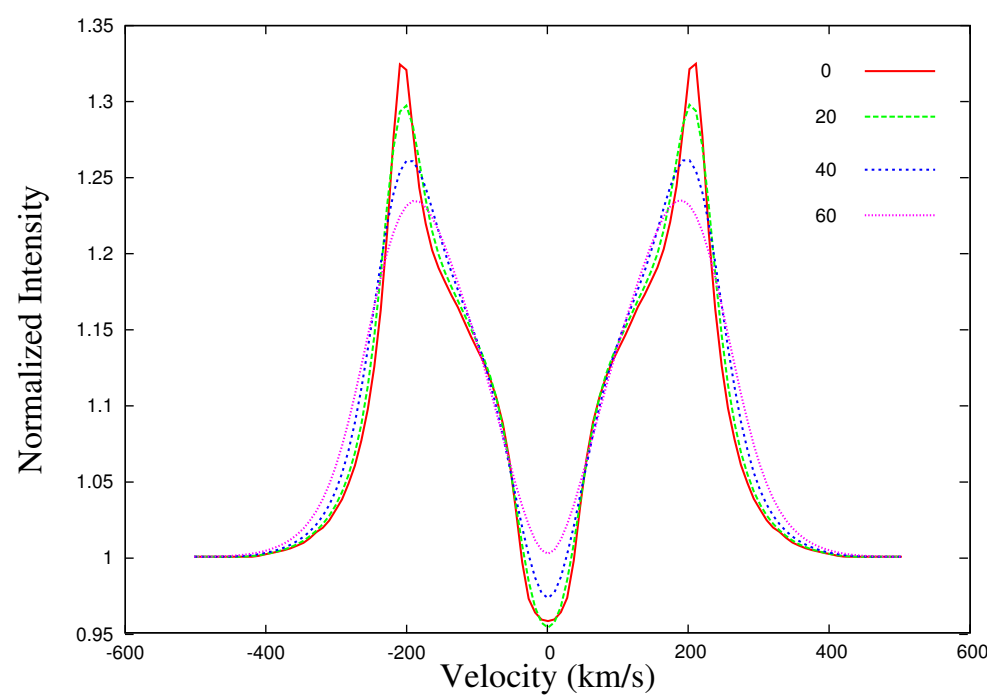

Figure 9. Effect of increasing the microturbulence in the disc from 0 to $60 \mathrm{~km} \mathrm{~s}^{-1}$. 


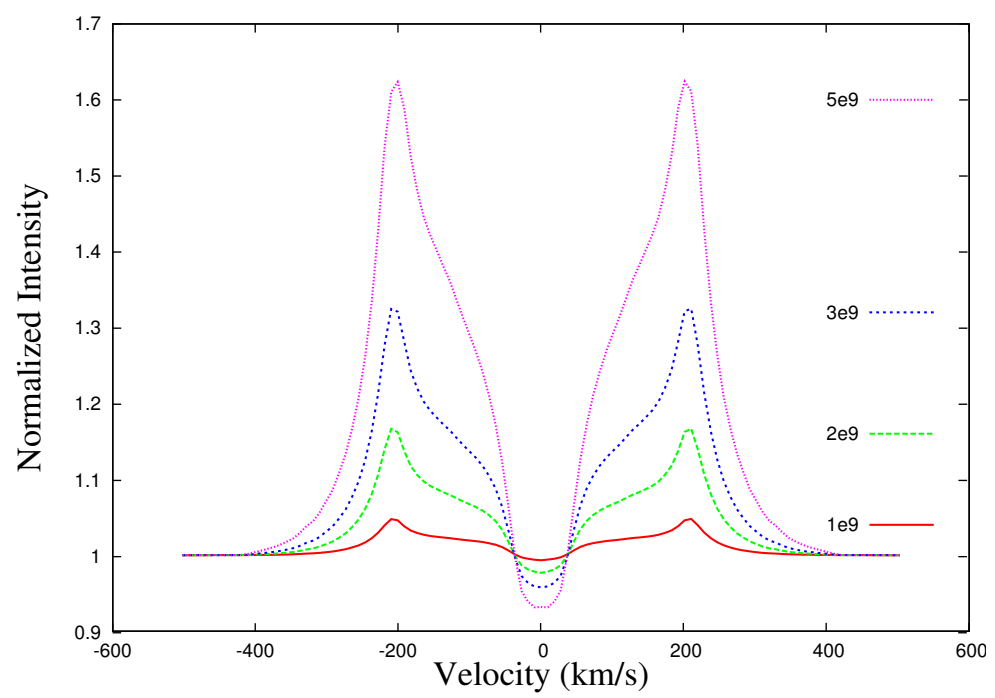

Figure 10. Effect of varying the density and the electron number density of the disc. $n_{\mathrm{e}}$ varies from $1 \times 10^{9}$ to $5 \times 10^{9} \mathrm{~cm}^{-3}, \rho \sim n_{\mathrm{e}}$.

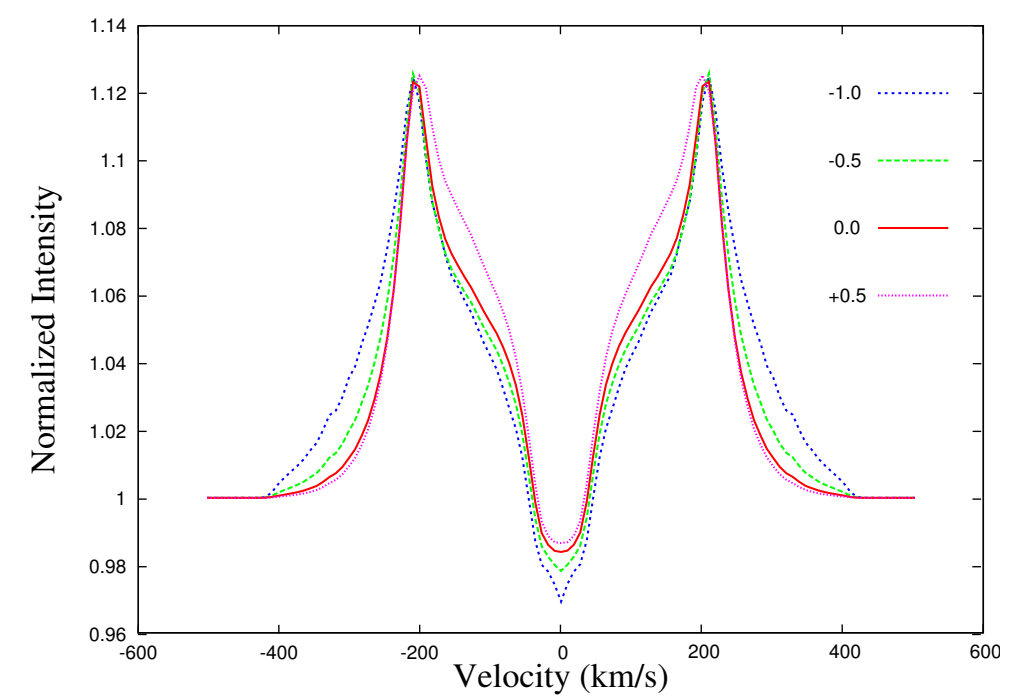

Figure 11. Effect of varying the exponent edendc $\equiv \eta$ of the power law behavior of the densities $n_{\mathrm{e}} \sim \rho \sim r^{\eta}$ in the disc, $\eta=-1.0,-0.5,0 .,+0.5$. Spectra are normalized to the same emission peak strength. 


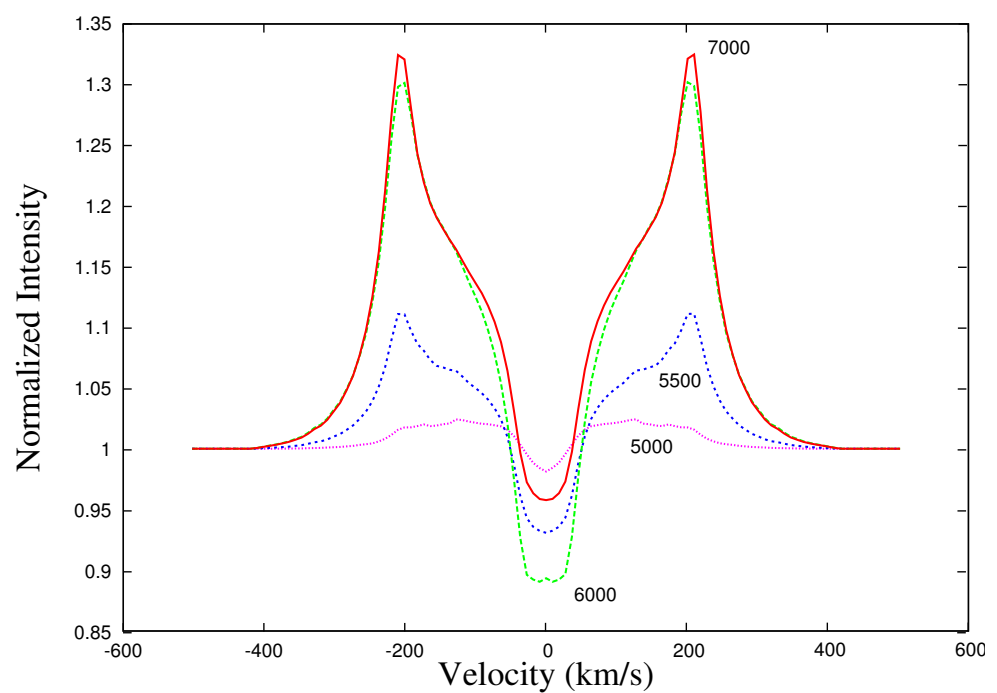

Figure 12. Effect of varying temperature of the disc from $5000 \mathrm{~K}$ to $7000 \mathrm{~K}$.

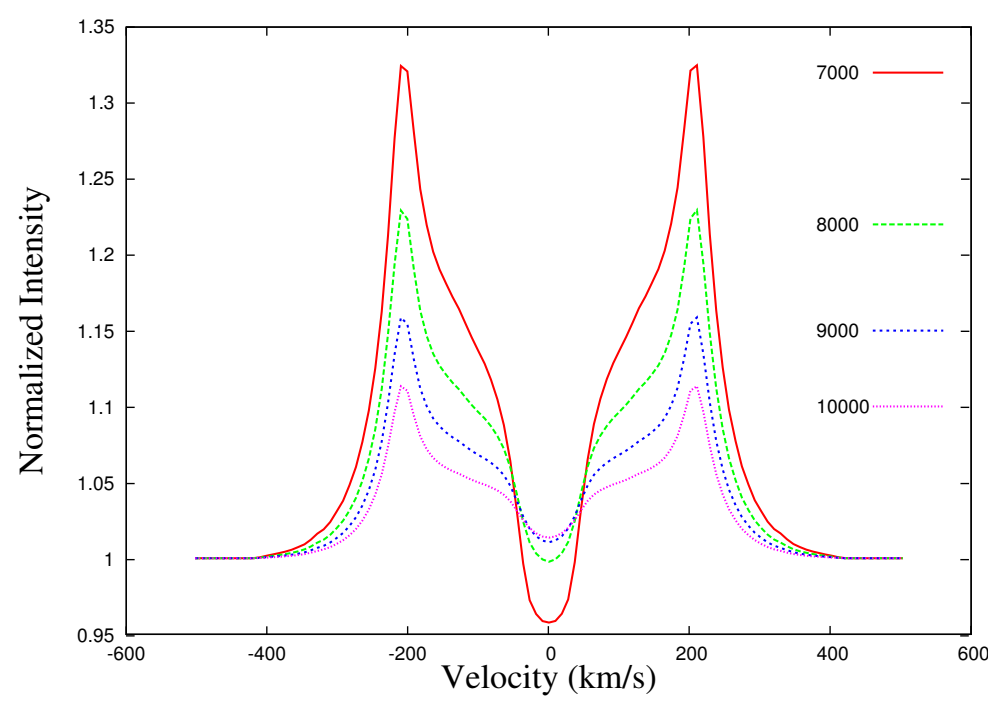

Figure 13. Effect of varying temperature of the disc from $7000 \mathrm{~K}$ to $10000 \mathrm{~K}$. 


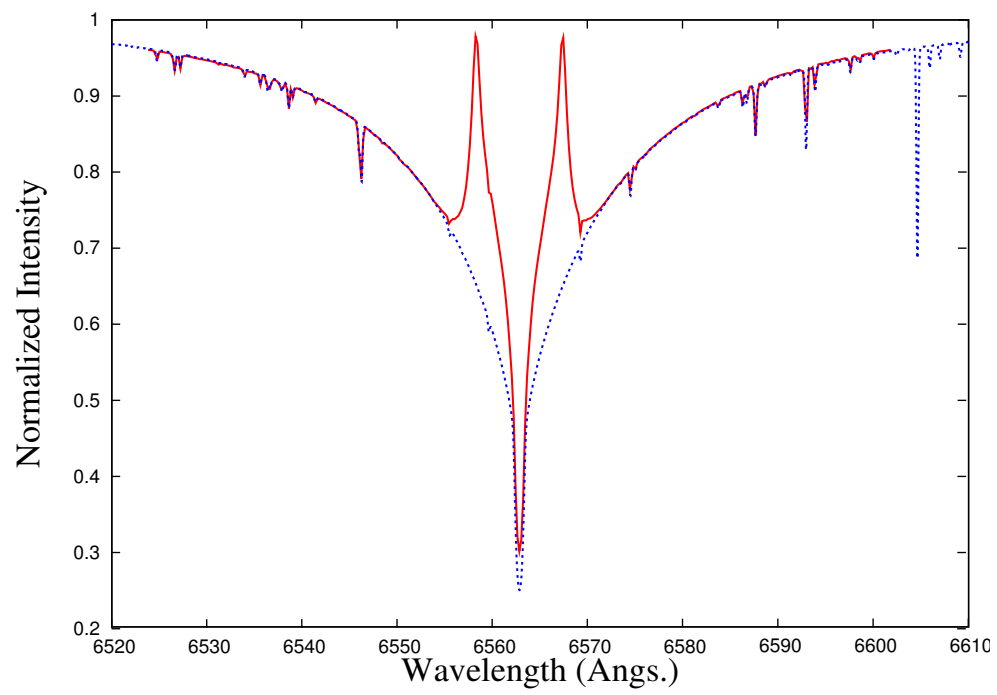

Figure 14. Synthetic spectrum of the primary (dashed line), composite synthetic spectrum of the primary with disc (solid line).

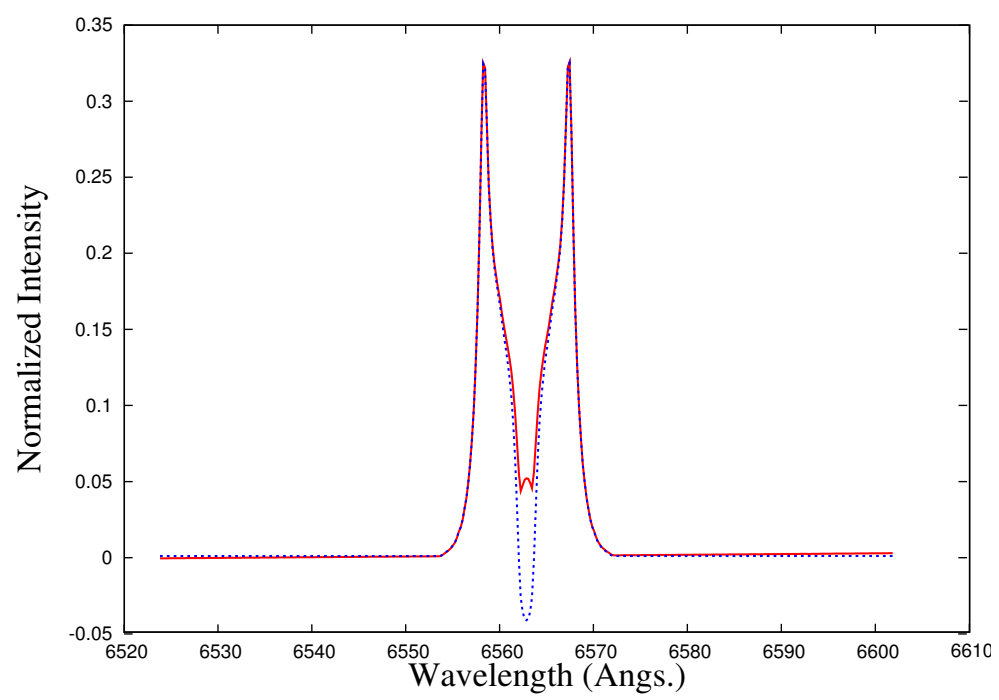

Figure 15. Synthetic difference profiles calculated when primary has its own intrinsic spectrum (solid line) or when primary was treated as a blackbody (dashed line). 


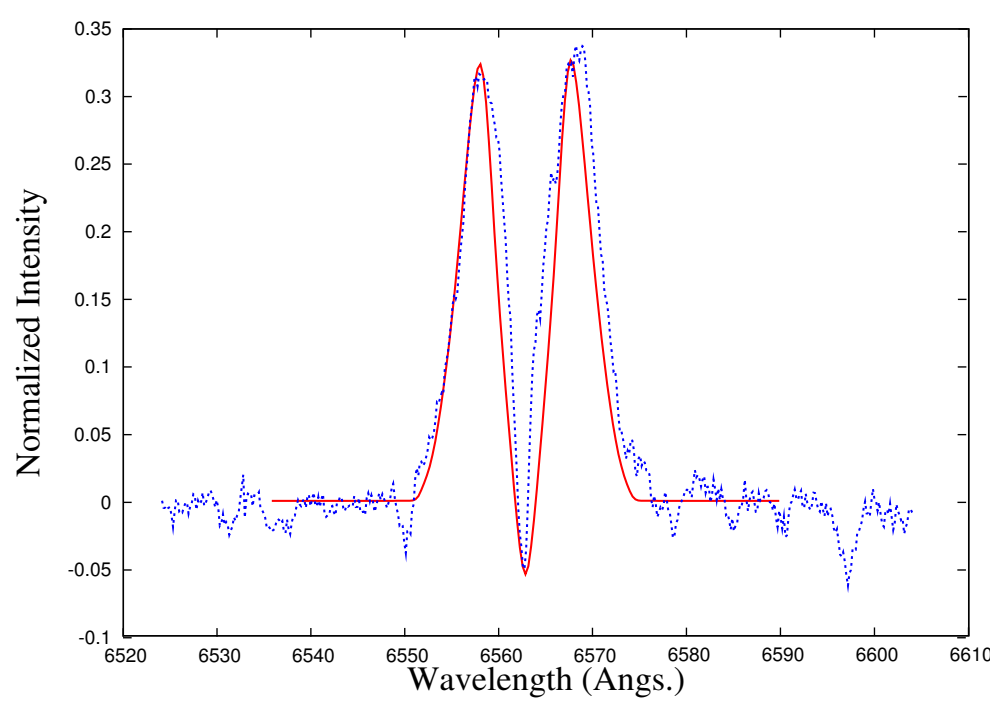

Figure 16. Observed difference profiles (dashed line) compared to synthetic difference profiles calculated when primary was treated as a blackbody (solid line).

\section{Acknowledgements}

We would like to thank Drs. J. Kubát and A. Skopal for their comments and suggestions. JB gratefully acknowledges grant support from Penn State University and thanks Drs. K. Getman, M. Tsujimoto, and the department computer staff for their assistance with computer related problems. This research was supported by the NSF-NATO fellowship (NSF DGE-0312144) and partly by the VEGA grant No. 3014 from the Slovak Academy of Sciences and the Science and Technology Assistance Agency under the contract No. 51-000802. This study made use of the Vienna Atomic Line Data Base (VALD) services.

\section{References}

Albright, G.E., Richards, M.T. 1996, ApJ 459, L99

Bradstreet, D.H., Steelman, D.P. 2002, Bull. Am. Astron. Soc. 34, 1224

Budaj, J., Dworetsky, M.M. 2002, MNRAS 337, 1340

Cherepashchuk, A.M., Eaton, J.A., Khaliullin, K.F. 1984, ApJ 281, 774

Djurasevic, G. 1992, ApESSS 197, 17

Drechsel, H., Haas, S., Lorenz, R., Mayer, P. 1994, A\&GA 284, 853

Etzel, P.B. 1988, AJ 95, 1204

Hadrava, P. 1997, A\&SAS 122, 581

Hill, G. 1979, Publ. Dom. Ap. Obs. Victoria 15, 297

Horne, K., Marsh, T.R. 1986, MNRAS 218, 761

Hubeny, I. 1988, Comput. Phys. Comm. 52, 103

Hubeny, I., Lanz, T. 1992, A\&A 262, 501

Hubeny, I., Lanz, T. 1995, ApJ 439, 875

Hubeny, I., Lanz, T., Jeffery, C.S. 1994, in C.S. Jeffery (ed.), Newsletter on Analysis of Astronomical spectra No.20 (CCP7; St. Andrews St. Andrews Univ.), 30

Krtička, J. 1998, in Dušek J. \& Zejda M. (eds.), Proc. 20th Stellar Conf., Nicholas Copernicus Observatory and Planetarium, Brno, p. 73

Krtička, J., Kubát, J. 2002, A\&A 388, 531

Kubát, J. 2001, A\&̊A 366, 210

Kulkarni, A.G., Abhyankar, K.D. 1980, A\&SS 67, 205 
Kurucz, R.L. 1990, Trans. IAU, XXB, 168 (CD-ROM 23)

Kurucz, R.L. 1993, ATLAS9 Stellar Atmosphere Programs and 2 km/s grid (Kurucz CD-ROM 13)

la Dous, C. 1989, A\&\&A 211, 131

Long, K.S., Knigge, C. 2002, ApJ 579, 725

Lucy, L.B. $1968, A p J$ 153, 877

Mochnacki, S.W., Doughty, N.A. 1972, MNRAS 156, 51

Orosz, J.A., Wade, R.A. 2003, ApJ 593, 1032

Peters, G.J. 1989, Space Sci. Rev., 50, 9

Peters, G.J., Polidan, R.S. 1998, ApJ 500, L17

Piskunov, N.E. 1992, in Yu.V. Glagolevskij \& I.I. Romanyuk (eds.), Stellar Magnetism, Nauka, Sankt Petersburg, 92

Plavec, M.J., Polidan, R.S. 1976, in P. Eggleton, S. Mitton \& J. Whelan (eds.), IAU Symp. 73, Structure and Evolution of Close Binary Systems, (DordrechtReidel), 289

Plavec, M.J. 1988, AJ 96, 755

Press, W.H., Flannery, B.P., Teukolsky, S.A., Vetterling, W.T. 1986, Numerical Recipes, Cambridge Univ. Press, Cambridge

Pribulla, T. 2004, in Hilditch, R., H. Hensberge and K. Pavlovski (eds.), Spectroscopically and spatially Resolving the Components of Close Binary Stars, ASP Conf. series., in press

Proga, D., Kallman, T.R., Drew, J.E., Hartley, L.E. 2002, ApJ 572, 382

Richards, M.T., Albright, G.E. 1999, ApJS 123, 537

Richards, M.T., Ratliff, M.A. 1998, ApJ 493, 326

Rucinski, S.W. 1973, Acta Astr. 23, 79

Rybicki, G.B., Hummer, D.G. 1983, ApJ 274, 380

Smith, K.C., Dworetsky, M.M. 1988, in S.J. Adelman \& T. Lanz (eds.), Elemental Abundance Analyses. Institut d'Astronomie de l'Univ. de Lausanne, Switzerland, p. 32

Van Hamme, W., Wilson, R.E. 1993, MNRAS 262, 220

Vinkó, J., Hegedüs, T., Hendry, D. 1996, MNRAS 280, 489

Wade, R.A., Hubeny, I. 1998, ApJ 509, 350

Wilson, R.E., Devinney, E.J. 1971, ApJ 166, 605

Zhang, E.-H., Robinson, E.L., Nather, R.E. 1986, ApJ 305, 740 